\title{
Factors Associated with Screening Baby Boomers for Hepatitis C Virus Infection Among Primary Care Providers: a Retrospective Analysis
}

J Gen Intern Med 36(11):3584-6

DOI: $10.1007 / \mathrm{s} 11606-020-06371-3$

(C) Society of General Internal Medicine 2021

\section{INTRODUCTION}

Hepatitis $\mathrm{C}$ virus (HCV) infection is a leading cause of hepatocellular carcinoma (HCC), a cancer that is increasing in incidence. ${ }^{1}$ Approximately 2.4 million Americans are chronically infected with $\mathrm{HCV}$, but most are unaware of their infection. ${ }^{2}$ Multiple public health organizations recommend a one-time test of all patients born 1945-1965 (i.e., baby boomers), in addition to risk-based testing, and a recent addition of a one-time test for all adults ages $18-79 .{ }^{3}$ Despite screening recommendations and effective treatments, $\mathrm{HCV}$ screening remains low. ${ }^{4}$ Understanding factors associated with screening asymptomatic baby boomers is essential to developing interventions to achieve universal screening.

Primary care providers ( $\mathrm{PCPs}$ ) provide routine, preventive health care, and are most likely to screen asymptomatic patients for HCV. This study assessed (1) the frequency of baby boomer HCV screening orders, and (2) provider characteristics associated with ordering HCV screening over a three-year period.

\section{METHODS}

We conducted a retrospective study examining electronic health records (EHR) for patient visits to a PCP from 8/1/ 2015 to 7/31/2018, described elsewhere. ${ }^{4}$ EHR data included providers' primary care specialty (i.e., Family Medicine, Internal Medicine, Internal Medicine/Pediatrics, Obstetrics/Gynecology), National Provider Identifier (NPI), and HCV screening orders. Provider demographic characteristics (e.g., age, gender) were ascertained using the NPI. Patients were included if they were baby boomers, without evidence of prior $\mathrm{HCV}$ screening, and had a visit with a PCP.

All visits with an $\mathrm{HCV}$-screening eligible baby boomer patient were identified. Screening opportunities were defined as EHR recorded visits to a PCP by unscreened baby boomers.

Received July 13, 2020

Accepted November 25, 2020

Published online January 14, 2021
$\mathrm{HCV}$ antibody screening tests ordered during these encounters were identified and coded as binary (yes/no). The percentage of opportunities where a screen was actually ordered was compared between provider specialty using ANOVA. We then stratified by provider specialty and assessed screening rates by provider age, sex, country in which they attended medical school, and years practicing medicine. Chi-square and Pearson's correlations assessed whether provider characteristics were associated with screening orders.

\section{RESULTS}

The sample included 68 PCPs who engaged in 20,698 (median = 186) opportunities to order an HCV screen with eligible baby boomer patients. The majority $(n=47 ; 69.2 \%)$ were Internal Medicine or Internal Medicine/Pediatrics. Approximately half of providers were female $(n=33 ; 48.5 \%)$, had an average age of 40.7 years, and an average of 12.6 years practicing medicine (Table 1).

\section{HCV Screening by Provider Type}

Overall, providers ordered HCV screening during an average of $9.4 \%$ ( $\mathrm{SD}=15.6)$ of their opportunities with eligible baby boomer patients. On average, Family Medicine providers ordered HCV screening during $29.4 \%$ of their opportunities compared to $\mathrm{OB} / \mathrm{GYNs}$ who ordered screening less than $1 \%$ of their opportunities ( $p=0.0064$; Fig. 1).

Almost no provider characteristics were significantly associated with HCV test orders (Table 1). Among Internal Medicine/Pediatrics providers, years practicing medicine was positively associated with HCV screening tests ordered $(p=$ 0.03 ). Comparable correlations were observed in the Family Medicine group, but the sample size was not sufficient for this correlation to be statistically significant. There were no observed associations with HCV screening by provider sex ( $p \mathrm{~s}=$ $0.07-0.98)$, medical school country $(p s=0.42-0.59$ ), or age $(p \mathrm{~s}=0.06-0.94)$.

\section{DISCUSSION}

PCPs were the focus of this study as they provide routine, preventive health care, and are the most likely to order HCV screening for asymptomatic patients. Overall, HCV screening 
Table 1 Provider Characteristics and Average Rate of HCV Screening by Provider Specialty $(N=68)$

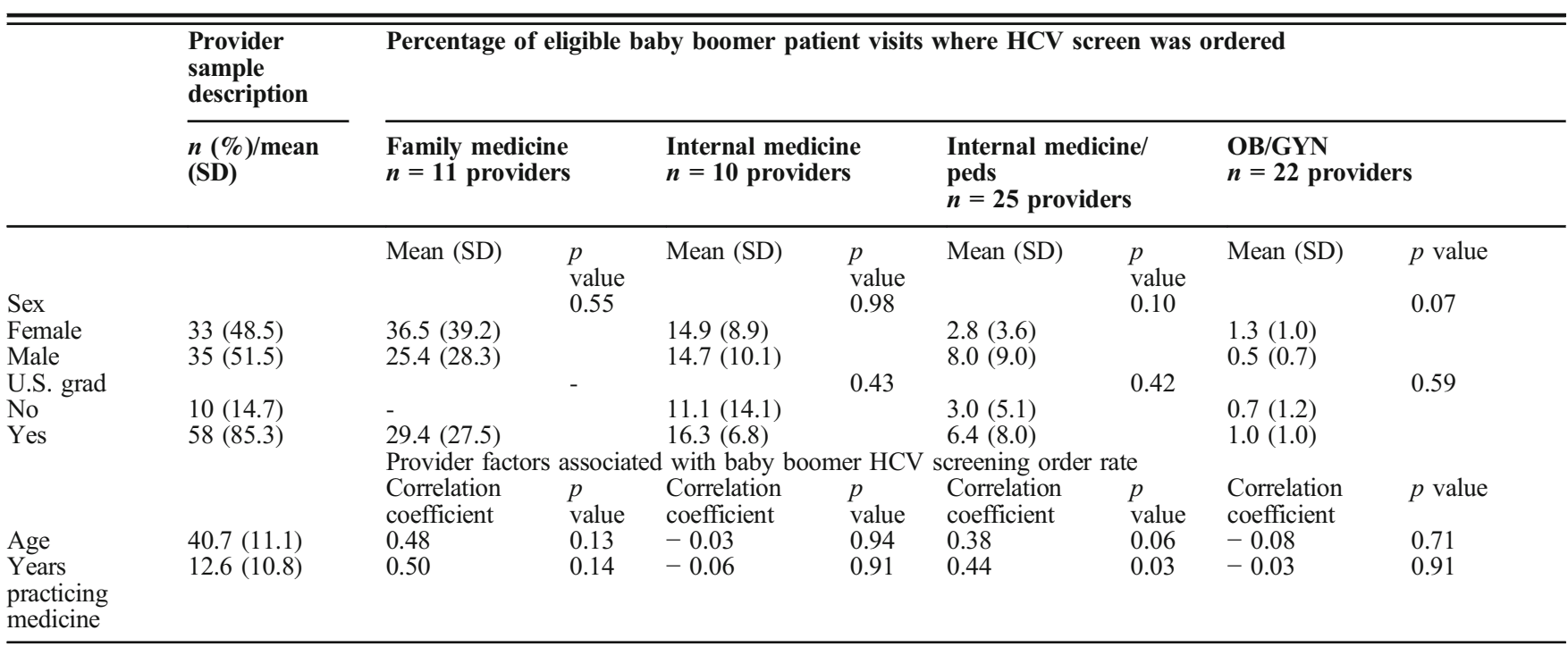

Mean percent of HCV screening for each provider was calculated by (number of eligible baby boomers screen orders)/(number of eligible baby boomer patient visits)

was ordered at a low rate, with Family Medicine providers and providers with more years in practice having the highest rates, although all fall far below national guidelines for universal HCV screening among baby boomers. OB/GYNs on average ordered screens during less than $1 \%$ of their opportunities. This is concerning because $\sim 15 \%$ of women obtain primary care services from their reproductive health care provider, and screening is now recommended during pregnancy., ${ }^{3,5}$ Thus, the opportunity to be screened for HCV is lost for a significant proportion of women.

The lack of association between physician characteristics and $\mathrm{HCV}$ ordering rates, coupled with overall low rates of screening, supports the need for a broad-based strategy for all PCPs to increase ordering HCV screening among baby

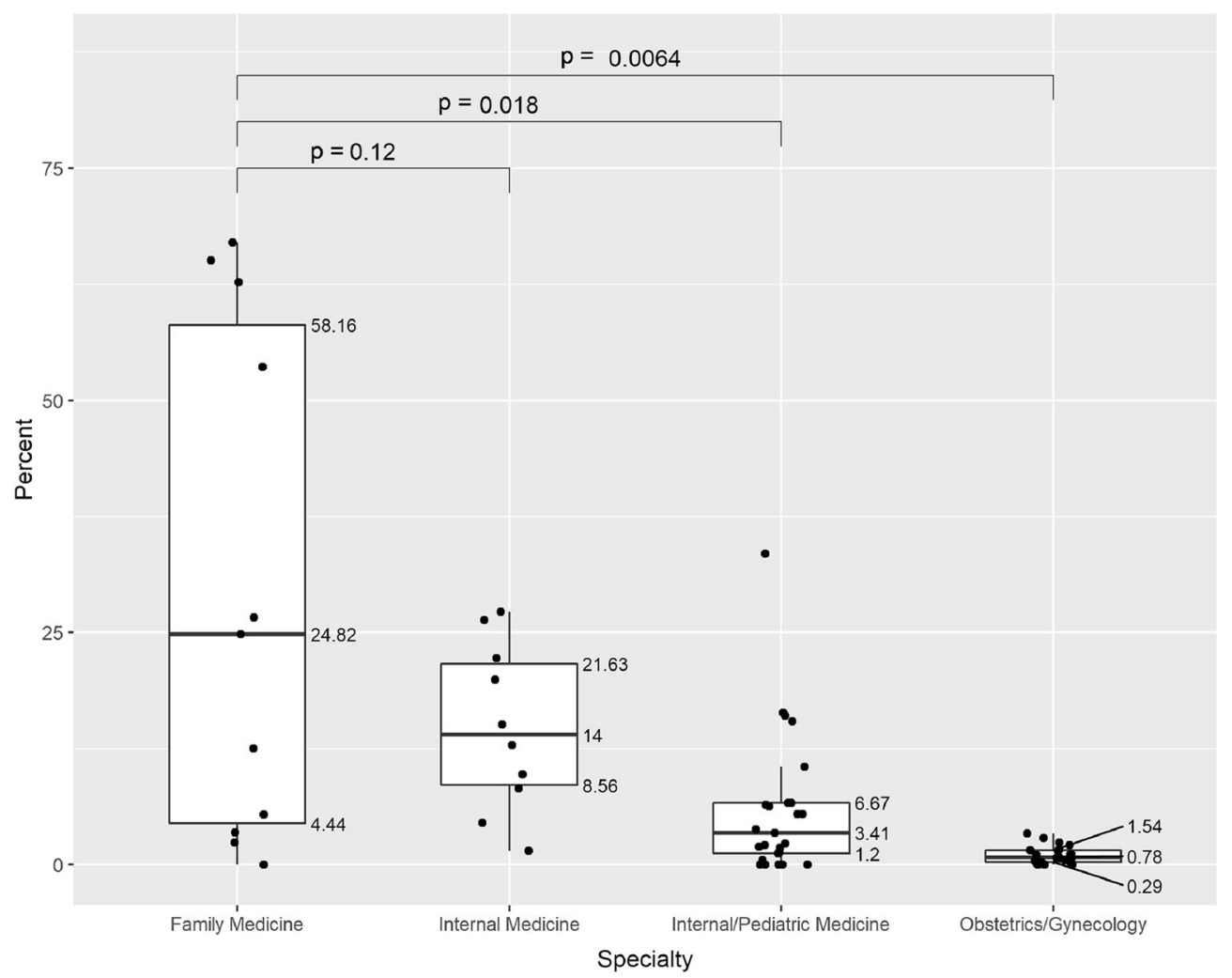

Figure 1 Percent of eligible baby boomer visits ${ }^{\mathrm{a}}$ where $\mathrm{HCV}$ screening was ordered grouped by primary care specialty ${ }^{\mathrm{b}}$. ${ }^{\mathrm{a}}$ Eligible visits were EHR recorded visits with a primary care physician. ${ }^{b}$ First quartile, median, and third quartile reported. 
boomers. Such strategies include best practice alerts in the EHR or other quality improvement initiatives, which have been demonstrated to significantly increase $\mathrm{HCV}$ screening. ${ }^{6}$ If successful, these approaches can reduce the burden of $\mathrm{HCV}$ related diseases among baby boomers and other populations, including all adults 18-79 years old who are newly recommended for HCV screening. ${ }^{2}$

Acknowledgments: Contributors: The authors would like to acknowledge Wenyi Fan for assisting in making the figure for the manuscript and William Stewart for assisting in data acquisition and working with the electronic health record.

Monica L. Kasting, $\mathrm{Ph} \mathrm{D}^{1,2}$

Anna R. Giuliano, $\mathrm{PhD}^{3,4}$

Richard R. Reich, $P h D^{5}$

Julie Rathwell, $M_{P H}{ }^{3,4}$

Richard G. Roetzheim, $M D^{6,7}$

Susan T. Vadaparampil, $\mathrm{Ph} \mathrm{D}^{4,6,8}$

${ }^{1}$ Department of Public Health, Purdue University, West Lafayette, IN, USA

${ }^{2}$ Cancer Prevention and Control Program, Indiana University Simon Comprehensive Cancer Center,

Indianapolis, IN, USA

${ }^{3}$ Department of Cancer Epidemiology, Moffitt Cancer Center,

Tampa, FL, USA

${ }^{4}$ Center for Immunization and Infection Research in Cancer, Moffitt Cancer Center,

Tampa, FL, USA

${ }^{5}$ Biostatistics and Bioinformatics Shared Resource, Moffitt Cancer Center,

Tampa, FL, USA

${ }^{6}$ Department of Health Outcomes and Behavior, Moffitt Cancer Center,

Tampa, FL, USA

${ }^{7}$ Department of Family Medicine, University of South Florida,

Tampa, FL, USA
${ }^{8}$ Department of Oncologic Sciences, University of South Florida,

Tampa, FL, USA

Corresponding Author: Susan T. Vadaparampil, PhD; Department of Health Outcomes and Behavior, Moffitt Cancer Center, Tampa, FL, USA (e-mail: susan.vadaparampil@moffitt.org).

Funding This work was supported, in part, by a 2016 Moffitt Cancer Center Team Science Award (PIs: Giuliano and Vadaparampil) and also by the Biostatistics and Bioinformatics Shared Resource at the $H$. Lee Moffitt Cancer Center \& Research Institute, an NCI-designated Comprehensive Cancer Center (P30-CA076292; PI: Cleveland).

\section{Compliance with Ethical Standards:}

Conflict of Interest: Dr. Vadaparampil has received research funding from Gilead Sciences. The other authors have no conflicts of interest to declare.

\section{REFERENCES}

1. Cronin KA, Lake AJ, Scott S, et al. Annual report to the nation on the status of cancer, part I: National cancer statistics. Cancer. 2018;124(13):2785-2800.

2. Centers for Disease Control and Prevention. Viral Hepatitis: Hepatitis C. Available at: https://www.cdc.gov/hepatitis/hcv/index.htm. Accessed Sept 5, 2019.

3. U.S. Preventive Services Task Force. Screening for Hepatitis C Virus Infection in Adolescents and Adults: US Preventive Services Task Force Recommendation Statement. JAMA. 2020;323(10):970-975. doi:https:// doi.org/10.1001/jama.2020.1123.

4. Kasting ML, Giuliano AR, Reich RR, et al. Electronic medical recordverified hepatitis $\mathrm{C}$ virus screening in a large health system. Cancer Med 2019;8(10):4555-4564.

5. Hall KS, Harris LH, Dalton VK. women's preferred sources for primary and mental health care: Implications for reproductive health providers. Womens Health Issues 2017;27(2):196-205.

6. Yeboah-Korang A, Beig MI, Khan MQ, et al. Hepatitis C screening in commercially insured U.S. birth-cohort patients: Factors associated with testing and effect of an EMR-based screening alert. J Transl Int Med 2018;6(2):82-89.

Publisher's Note: Springer Nature remains neutral with regard to jurisdictional claims in published maps and institutional affiliations. 